\title{
Experimental design and numerical investigation of a photoacoustic sensor for a low-power, continuous-wave, laser-based frequency-domain photoacoustic microscopy
}

Krishnan Sathiyamoorthy

Michael C. Kolios 


\title{
Experimental design and numerical investigation of a photoacoustic sensor for a low-power, continuous-wave, laser-based frequency-domain photoacoustic microscopy
}

\author{
Krishnan Sathiyamoorthy ${ }^{a, b, c}$ and Michael C. Kolios ${ }^{a, b, c}$ \\ ${ }^{a}$ Ryerson University, Department of Physics, Toronto, Ontario, Canada \\ 'Institute for Biomedical Engineering, Science, and Technology (iBEST), a partnership between Ryerson University and St. Michael's Hospital, \\ Toronto, Canada \\ ${ }^{\circ}$ Keenan Research Centre for Biomedical Science, St. Michael's Hospital, Toronto, Ontario, Canada
}

\begin{abstract}
We have developed a photoacoustic (PA) sensor using a low-power, continuous- wave laser and a $\mathrm{kHz}$-range microphone. The sensor is simple, flexible, cost-effective, and compatible with commercial optical microscopes. The sensor enables noncontact PA measurements through air, whereas most current existing PA techniques require an acoustic coupling liquid for detection. The PA sensor has three main components: one is the chamber that holds the sample, the second is a resonator column used to amplify the weak PA signals generated within the sample chamber, and the third is a microphone at the end of the resonator column to detect the amplified signals. The chamber size was designed to be $8 \mathrm{~mm} \times 3 \mathrm{~mm}$ as the thermal diffusion length and viscous-thermal damping of air at room pressure and temperature are 2 and $1 \mathrm{~mm}$, respectively. We numerically and experimentally examined the effect of the resonator column size on the frequency response of the PA sensor. The quality factor decreased significantly when the sample chamber size was reduced from $4 \mathrm{~mm} \times 3 \mathrm{~mm}$ to $2 \mathrm{~mm} \times 3 \mathrm{~mm}$ due to thermos-viscous damping of the air. The quality factor decreased by $27 \%$, demonstrating the need for optimal design for the sample chamber and resonator column size. The system exhibited noise equivalent molecular sensitivity (NEM) per unit bandwidth (NEM/ $\sqrt{ } \Delta f$ ) of $\sim 19,966 \mathrm{~Hz}^{-1 / 2}$ or $33 \times 10^{-21}$ mol or 33 zeptomol, which is an improvement of 2.2 times compared to the previous system design. This PA sensor has the potential for noncontact high-resolution PA imaging of materials without the need for coupling fluids. ๑ The Authors. Published by SPIE under a Creative Commons Attribution 4.0 Unported License. Distribution or reproduction of this work in whole or in part requires full attribution of the original publication, including its DOI. [DOI: 10.1117/1.JBO.24.12.121912]
\end{abstract}

Keywords: microscope; photoacoustic; lasers; COMSOL; resonator; microphone; biomedical.

Paper 190181SSRR received Jun. 3, 2019; accepted for publication Oct. 1, 2019; published online Oct. 31, 2019.

\section{Introduction}

The photoacoustic (PA) effect was first observed in the year 1880 by Alexander Graham Bell. ${ }^{1}$ The PA effect was used in spectroscopy techniques throughout the mid-1900s, but the invention of the laser has significantly changed the paradigm of the PA technique because of the improved sensitivity provided by pulsed laser excitation. ${ }^{2-13}$

Photoacoustic microscopy (PAM) is a hybrid imaging modality that uses an optical technique for excitation and an acoustic technique for detection. The general principle is based on the generation of acoustic waves due to transient pressure variation caused by means of absorption of a short (typically nanosecond) light pulse. As high-resolution deep tissue imaging is possible using acoustic resolution PA imaging, ${ }^{14-17}$ PA imaging finds several potential applications providing anatomical, ${ }^{18}$ functional, ${ }^{14}$ molecular, ${ }^{14}$ flow dynamic,,${ }^{15}$ and metabolic contrast in vivo. ${ }^{15}$

Microscopy applications can use either acoustic resolution PAM (AR-PAM) or optical-resolution PAM (OR-PAM), or a combination of both, depending on the application and imaging target. ${ }^{19}$ The lateral resolution of AR-PAM depends on the acoustic focus of the transducer which is of the order of tens

*Address all correspondence to Michael C. Kolios, E-mail: mkolios@ ryerson.ca of micrometers with a maximum imaging depth of several millimeters whereas the lateral resolution of OR-PAM depends on the diffraction limit of the optical lens with a maximum possible imaging depth of about $1.2 \mathrm{~mm} \cdot{ }^{20,21}$ Hence, OR-PAM has been used to image biological samples and small animals with various endogenous or exogenous contrasts ${ }^{20}$ whereas AR-PAM finds both preclinical and clinical applications as deep imaging of biological tissues is possible at lower ultrasound frequencies. ${ }^{22,23}$

Researchers are also interested in noncontact PA systems for remote sensing. Most of the noncontact PA systems are based on an interferometric principle. ${ }^{24-28}$ However, interferometric techniques can be complex, bulky, expensive, and difficult to integrate with the existing optical microscopes. ${ }^{24,29}$ A Fabry-Perot sensor is another option, but it is still a contact technique as the Fabry-Perot sensor has to be coupled to the sample..$^{10,30}$ Researchers have investigated using a fiber sensor, which also required expensive focusing and collection optics. Optical beam deflection techniques are another simple method for PA wave detection. However, this approach requires two lasers for pumping and probing and a large footprint area for the set up because the probe beam needs to travel a relatively long distance (for increasing deflection angle) before detection to improve sensitivity. $^{31,32}$ There are also other air-coupled transducers available, but air-coupled transducers are unfocused, bulky, and exhibit poor SNR, which makes imaging more complicated. ${ }^{33}$ 
Conventional PA imaging systems require expensive ultrafast lasers with nanosecond pulse widths, an ultrasound transducer (or optical-based ultrasound detection), and an aqueous coupling medium for the PA waves to propagate through. ${ }^{10,17,19}$ Wide clinical adoption has been hindered due to its expense, technical complexity, and cumbersome size. Considerable efforts have been made to use low-cost continuous-wave $(\mathrm{CW})$ lasers instead of ultrafast laser for PA imaging since $1990 .{ }^{34-36}$ Petschke and La Rivière ${ }^{35}$ theoretically investigated the possibility of using chirped $\mathrm{CW}$ diode lasers, which would exhibit 20 to $30 \mathrm{~dB}$ lower SNR than the typical pulsed laser-based systems, but its compactness and relatively low cost could potentially outweigh the lower SNR in select applications. $^{34,35,37}$

Recently, we performed noncontact PA studies using a lowpower CW laser. ${ }^{38}$ The weak PA signal generated by the lowpower $\mathrm{CW}$ laser was amplified using an acoustic resonator and measured using a $\mathrm{kHz}$ microphone. The system was studied on a red blood cell (RBC) and found exhibited a noise-equivalent detection sensitivity of 43,887 hemoglobin molecules $(72.88 \times$ $10^{-21} \mathrm{~mol}$ or 72.88 zeptomole). The minimum pressure detectable limit (MPDL) of the sensor was $19.1 \mu \mathrm{Pa}$. The technique was inspired by low-power CW -ased PA spectroscopy as it exhibited trace gas detection sensitivity of parts per billion. ${ }^{3-5,11-13,39-47}$

The magnitude of the PA signal is proportional to the thermal diffusion length $(\mu)$ /thermal diffusivity of the sample which is a function of optical and thermal properties of the material. As the PA signal is generated by intensity-modulated light, the thermal diffusion length $(\mu)$ would become a function of the modulation frequency of the light and is given by

$\mu=\frac{k}{\pi \rho f c}$,

where $k$ is the thermal conductivity, $\rho$ is the density, $c$ is the specific heat, and $f$ is the modulation frequency. Figure 2(a) shows a schematic diagram of the most commonly used PA cell for spectroscopy studies. The sample is enclosed in an airtight metal chamber and excited over the surface. The PA signal is due to pressure or stress generated in the gas adjacent to the sample. This PA cell configuration may not be suitable for high-resolution optical resolution PAM because exciting the sample using a high-magnification $100 \times$ objective is difficult due to its shorter working distance. The typical working distance of a $100 \times$ objective is around $0.17 \mathrm{~mm}$ but the sample in the PA cell should be positioned at least $1 \mathrm{~mm}$ away from the optical window to avoid acoustic damping caused by the walls of the PA cell.

The PA sensor for high-resolution OR-PAM proposed in this work has a different configuration compared to those used for PA spectroscopy. Figure 2(b) shows the schematic diagram of the PA sensor. The sensor works in a transillumination configuration. The sample is excited at the sample-glass interface. The thermal properties of the glass substrate play a vital role in the PA generation as it might lead to heat conduction outside the chamber, which would result in a decrease in the PA signal. The influence of the glass on the PA generation due to the back and surface excitation of the sample (Fig. 2) was investigated. The thermal diffusion length and viscous-thermal damping of the air will also play important roles in the loss of PA signal depending on the size of the PA cell. The effects of these factors on the PA signal are numerically and experimentally investigated.

One advantage of the present PA sensor would be that it could be used in both epi- and trans-illumination microscopes. The earlier ones were bulky and sturdy because most of the sensors were made of metals. The developed sensor is thin, compact, inexpensive, and flexible. The system can be easily integrated/attached into any systems or object to study the optical properties. In addition, the configuration of the sensor would enable multimodal imaging such as optical, fluorescent, Raman, and PA imaging of samples. As the PA spectroscopy technique can be used to study samples in any state of matter (solid, liquid, and gas), the PA sensor is also expected to find similar applications in studying both solid and liquid samples. This study investigates the use of the PA sensor in OR-PAM applications, with a focus on cell design using numerical simulation techniques and experimental verification.

\section{Materials and Methods}

\subsection{Procurement of Blood}

Blood was collected by netCAD (Vancouver, Canada), the research division of Canadian Blood Services, under protocol 2013-001, which involves standard Canadian Blood Services collection and testing procedures of whole blood and delivery overnight at $4^{\circ} \mathrm{C}$, with continuous monitoring during shipment to ensure no temperature deviations occur. This procedure has been approved by the research ethics boards.

\subsection{Blood Sample Preparation}

The guidelines on handling the blood were followed in accordance with the recommendations of the International Society for Clinical Hemorheology and the European Society for Clinical Hemorheology and Microcirculation. The blood was centrifuged at room temperature at $2000 \times g$ for 6 min to separate the plasma and their Buffy coat. Isotonic phosphate-buffered saline (PBS) was used to wash RBCs two times. The centrifuged RBCs were then dispersed in PBS for the present studies. RBCs were smeared on microscope slides for studies.

\subsection{Experimental Setup}

A simple dual-modal optical/PAM system was developed to characterize the developed PA sensor (Fig. 1). The PA system is composed of an optical imaging system, a laser for PA excitation, a motorized translation stage to raster scan the sample, and the PA sensor. The optical imaging system was developed in inverted microscope configuration. The system contains an infinity-corrected $40 \times$ and 0.25 NA Olympus objective $(O)$, and a Ximea xiQ camera (Ximea). This configuration of this system was simpler than our earlier reported optical microscope system. ${ }^{38}$ The objective was moved to excite the sample in the earlier configuration, which required a complex optical configuration to keep the laser beam coaxial with the optic axis of the objective. The present configuration uses a fixed optical set-up in which the light beam is coupled directly to the stationary objective for excitation and the sample is moved.

A helium-neon laser (Spectra-Physics) with a beam diameter of $1 \mathrm{~mm}$ and a green laser (CrystaLaser) with a beam diameter $0.36 \mathrm{~mm}$ were the optical source of the PA system. The power of the laser was $4 \mathrm{~mW}$. The solid green line in Fig. 1 shows the laser path in the PA microscope system which was focused 


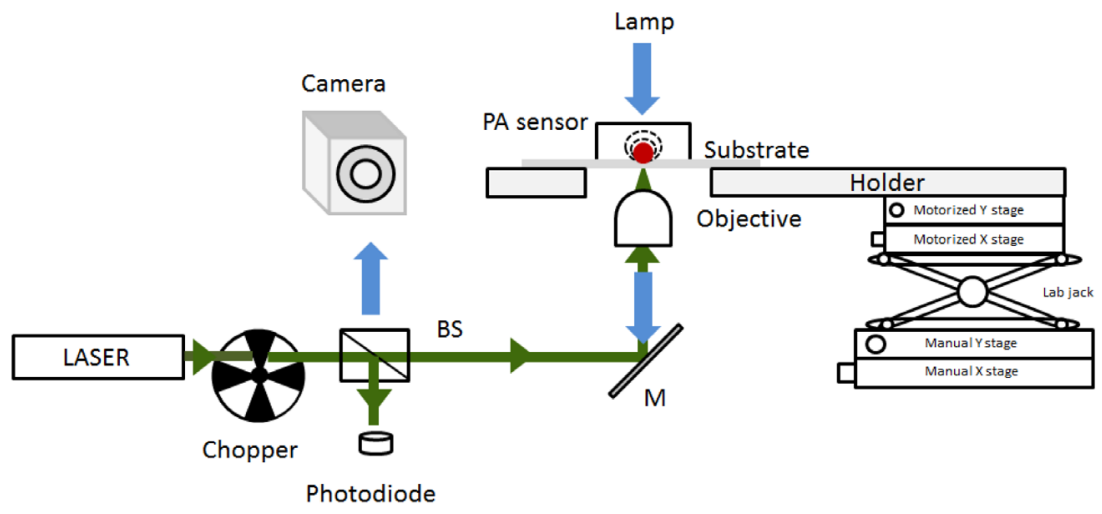

(a)

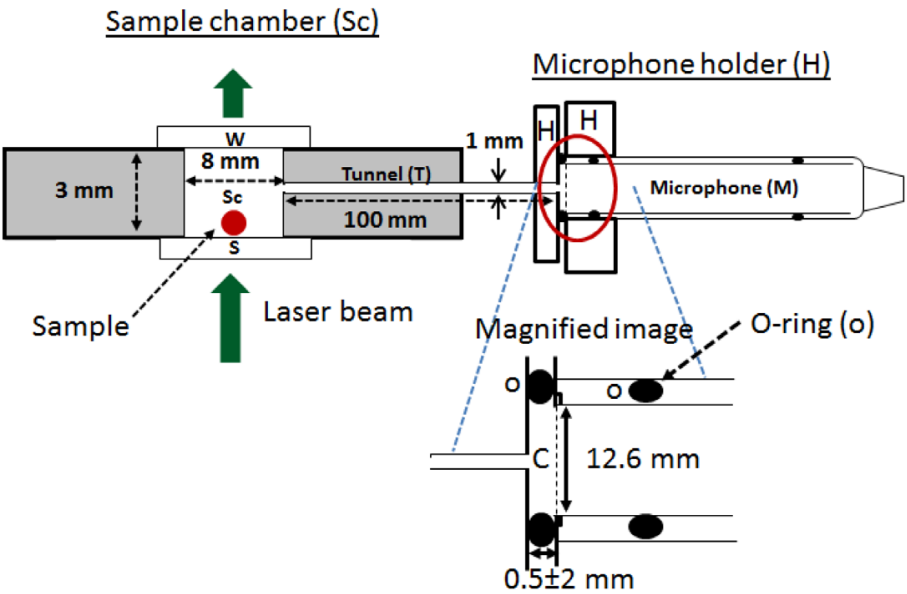

(b)

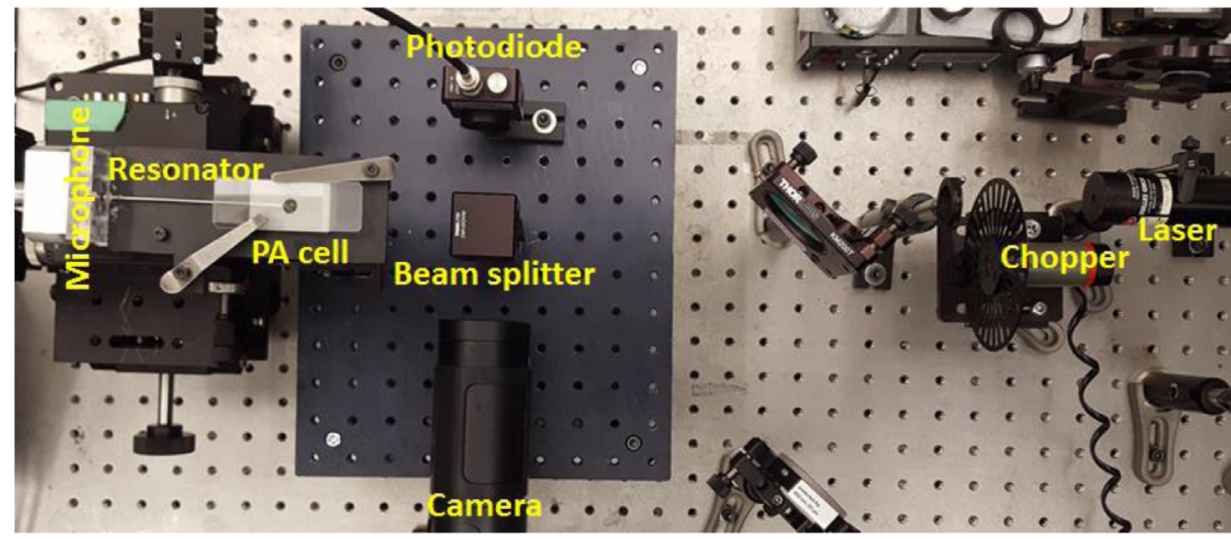

(c)

Fig. 1 A schematic diagram of the optical/PAM system. The solid green line and the blue arrows represent the laser beam path and polychromatic light beam direction in the PA and the optical microscope system, respectively. (b) The schematic diagram of PA sensor where $\mathrm{Sc}$ is the sample chamber, $\mathrm{S}$ is the glass substrate, $\mathrm{W}$ is the glass window, $\mathrm{T}$ is the tunnel, $\mathrm{C}$ is the coupler, $\mathrm{O}$ is the $\mathrm{O}$-ring, $\mathrm{H}$ is the holder, and $\mathrm{M}$ is the microphone. (c) The photograph of a top view of the system.

on the sample by an objective. A cube beam splitter was used to sample a small portion the input beam toward the photodiode (PD) for reference, and also used to direct the trans-illuminated LED light, which counterpropagates in the direction of the laser beam, from the sample to the camera positioned opposite to PD. The laser was modulated using a mechanical chopper (SR540,
Stanford Research Systems) to generate a pulsed output. The PA signal was measured using a lock-in amplifier (SR850, Stanford Research Systems). The system was automated using custom Matlab software (Mathworks).

The translation system consisted of manual $X-Y-Z$ stages (Thorlabs) for interrogating the sample and motorized linear 
$X-Y$ scanning stages from Zaber Technologies, for raster scanning the sample for PA imaging.

The measurement is made using a lock-in amplifier (SR850). The lock-in amplifier contains two main components: the preamplifier and the phase-sensitive detector (PSD). The input of the lock-in amplifier was first amplified before entering a PSD. The PSD detects the signal with respect to a reference by multiplication of the amplified PA signal with the lock-in reference signal produced by the crystal oscillator of lock-in amplifier in accordance with an input reference signal (in our case, the input reference signal is from the chopper). The output of the PSD is the product of two sine waves:

$$
\begin{aligned}
V_{\mathrm{psd}}= & V_{\mathrm{sig}} V_{L} \sin \left(\omega_{r} t+\theta_{\mathrm{sig}}\right) \sin \left(\omega_{L} t+\theta_{\text {ref }}\right) \\
= & \frac{1}{2} V_{\mathrm{sig}} V_{L} \cos \left(\left[\omega_{r}-\omega_{L}\right] t+\theta_{\text {sig }}-\theta_{\text {ref }}\right) \\
& -\frac{1}{2} V_{\mathrm{sig}} V_{L} \cos \left(\left[\omega_{r}+\omega_{L}\right] t+\theta_{\text {sig }}+\theta_{\text {ref }}\right),
\end{aligned}
$$

where $V_{\text {sig }}$ is the signal amplitude, $V_{L}$ is the reference amplitude, $\omega_{s}$ is the signal frequency, $\omega_{r}$ is the reference frequency, $\theta_{\text {sig }}$ is the signal phase, and $\theta_{\text {ref }}$ is the reference phase.

The SR850 is a dual-phase lock-in amplifier with a second PSD measure the signal at 90 deg out of phase with respect to the first PSD. The output is a contribution from both PSDs. The magnitude of the signal is given as

$R=\left(V_{\mathrm{psd} 1}^{2}+V_{\mathrm{psd} 2}^{2}\right)^{1 / 2}=V_{\mathrm{sig}}$.

In this work, first, the SNR of the PA system was investigated. The SNR mainly depends on the input configuration of the lock-in amplifier and the power of the laser. The system used low-power lasers. The lock-in amplifier response for the low-power laser was studied. Carbon black was used as the sample. The noise of the lock-in amplifier depends on filter bandwidth which is a function of signal integration time constant. The SNR of the system for an integration time of $10 \mathrm{~ms}, 300 \mathrm{~ms}, 1 \mathrm{~s}$, and $3 \mathrm{~s}$ was studied (please see the Supplemental Materials). A lock-in amplifier functional at a low pass filter setting/single RC filter with a frequency response of $6 \mathrm{~dB} /$ oct and a time constant $300 \mathrm{~ms}$ exhibited a low SNR of $3.31 \times 10^{-6}$ (please refer to the Supplemental Materials).

\subsection{Photoacoustic Sensor}

The PA sensor for high-resolution OR-PAM is shown in Fig. 2(b). The sample is excited at the sample-glass interface. The effect of glass on PA generation is studied experimentally. Two optical configurations (Fig. 2) are used for these studies. The sample is excited in front and backside in these configurations. The PA study using both configurations showed that the front surface excitation [Fig. 2(a)] exhibited about $74 \%$ greater signal compared to the backside excitation [Fig. 2(b)] (please see the Supplemental Materials).

A schematic of the PA sensor is shown in Fig. 1(b). The PA sensor is made up of acrylic photopolymers and aluminum. The PA sensor has two main compartments, one for holding the sample (called sample chamber, Sc) and the other a resonant column coupled to the microphone (M). The Sc is a hollow cylindrical of diameter $8 \mathrm{~mm}$ and a height $3 \mathrm{~mm}$. The sample chamber is built in acrylic photopolymers using a three-dimensional printer (ProJet 3510 HDPlus 3D printer). The top of the sample

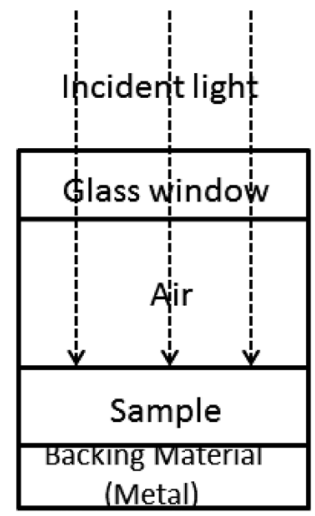

(a)

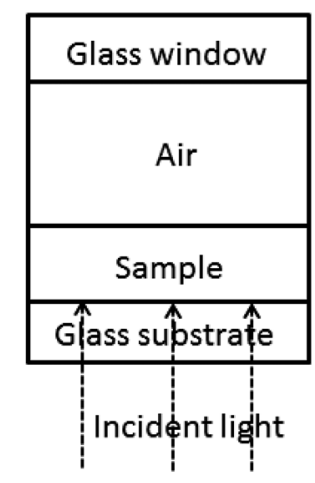

(b)
Fig. 2 A schematic diagram of (a) a typical PA cell configuration used for spectroscopy studies and (b) the PA cell used for the high-resolution PAM in these studies.

chamber is closed by an optical window (microscope slide), whereas the bottom of the chamber contains the sample (S) for study. A small resonant column (T) exists between the sample chamber and the microphone $(\mathrm{M})$ which is a cylinder of diameter $1 \mathrm{~mm}$ and varying length. A cylindrical coupler (C) of inner diameter $12.6 \mathrm{~mm}$ (equivalent to the diameter of the microphone) and height $0.5 \mathrm{~mm}$ is used to join the resonator column to the microphone because of a mismatch of their size (1 and $12.7 \mathrm{~mm}$, respectively). The microphone is housed in a specially designed rectangular holder $(\mathrm{H})$ with four holes at the corners for screws. The resonator, the coupler, and the microphone holder are made of aluminum. The microphone holder $\mathrm{H}$ is screwed airtight with respect to the coupler (C) using a rubber O-ring of inner diameter $16 \mathrm{~mm}$ and thickness $2 \mathrm{~mm}$ between them. The gap between the resonator and the microphone surface may vary between $0.5 \pm 0.2 \mathrm{~mm}$ due to the squeezing of rubber O-ring.

The resonator behaves like a resonance tube with an acoustic node formed at a closed-end (microphone end) and antinode formed at an open-end (sample chamber end). Resonance is observed when a standing acoustic mode is formed in the resonator. As the frequency of the acoustic mode is inversely proportional to the length of the resonator, the effect of the resonator length on the PA signal is studied. Resonator column lengths of 100, 200, and $400 \mathrm{~mm}$ were chosen for the study. The length was limited to $400 \mathrm{~mm}$ because of the size of the translation stage of most microscopes lies in this range.

The effect of the Sc size on the PA generation was also investigated. The sizes of the Sc chosen for these studies were $2 \mathrm{~mm} \times 3 \mathrm{~mm}, \quad 4 \mathrm{~mm} \times 3 \mathrm{~mm}, \quad 6 \mathrm{~mm} \times 3 \mathrm{~mm}$, and $8 \mathrm{~mm} \times 3 \mathrm{~mm}$. Sizes below $2 \mathrm{~mm}$ were not considered because of acoustic damping (the viscous thermal damping distance of air is $\sim 2 \mathrm{~mm}$ at $\mathrm{KHz}$ frequencies). A half-inch free-field microphone of type 4189 with a Type 2699 preamplifier (Brüel \& Kjær Sound \& Vibration Measurement, Pointe-Claire, Quebec, Canada) was used to measure the PA signal. The microphone has an almost flat frequency response from $50 \mathrm{~Hz}$ to $20 \mathrm{kHz}$ with a sensitivity of $50 \mathrm{mV} / \mathrm{Pa}$.

\subsection{Characterizing of a Photoacoustic Sensor}

The frequency response of the PA sensor was measured by modulating the output of the helium-neon laser using chopper 
from frequency 50 to $1100 \mathrm{~Hz}$. The frequency of the chopper was varied by changing its driver voltage by means of the potentiometer available in the system. ${ }^{48}$ The lock-in amplifier was programmed at low-pass filter setting with a frequency response of $\pm 6 \mathrm{~dB} /$ oct and time constant $300 \mathrm{~ms}$. The integration time can be increased at the cost of imaging speed to improve the $\mathrm{SNR}$ if the laser power is low.

\section{Photoacoustic Theory of a Cylindrical PA Sensor}

The sound field distribution in the cylindrical column representing the PA chamber is described by the Helmholtz equation ${ }^{49}$

$$
\begin{aligned}
& {\left[\frac{1}{r} \frac{\partial}{\partial r}\left(r \frac{\partial}{\partial r}\right)+\frac{1}{r^{2}} \frac{\partial^{2}}{\partial \varphi^{2}}+\frac{\partial^{2}}{\partial z^{2}}\right] p(r, \varphi, z, \omega)+k^{2} p(r, \varphi, z, \omega)} \\
& \quad=i \omega \frac{(\gamma-1)}{V_{C}} H(r, \varphi, z, \omega)
\end{aligned}
$$

where $V_{C}$ is the volume of the PA chamber, $\gamma$ is the ratio of the specific heats, $k$ is the wavenumber, and $H(r, \varphi, z, \omega)$ represents the Fourier transform of the heat source term $H(r, \varphi, z, t)$. Here, the heat source represents the sample heated by the laser. The sample with an absorption coefficient $\alpha$ and excited with light of intensity $I(r, \varphi, z, \omega)$ exhibits heating given by $H(r, \varphi, z, \omega)=\alpha I(r, \varphi, z, \omega)$, where is the amplitude $I(r, \varphi, z, \omega)$ is the Fourier transform of the laser intensity.

Solving the above inhomogeneous wave, Eq. (6) gives the acoustic modes supported by the cylindrical column ${ }^{50}$

$p(r, \varphi, z, \omega)=\sum_{j} A_{j}(\omega) p_{j}(r, \varphi, z)$,

where $A_{j}(\omega)$ is the amplitude of the acoustic mode $p_{j}(r, \varphi, z)$ and $\omega_{j}\left(\omega_{j}=c k_{j}\right.$ or $\left.2 \pi f_{j}=c k_{j}\right)$ is the eigenfrequency. The equation is solved by applying a sound hard wall boundary condition the normal derivative of the pressure $\left(\frac{\partial p}{\partial n}=0\right)$. The solution gives the supported eigenmodes of the PA sensor. The amplitude $A_{j}(\omega)$ and the frequency $f_{l m n}$ of the eigenmodes supported by the cylindrical PA sensor is given as

$A_{j}(\omega)=i \frac{A_{j} \omega}{\omega^{2}-\omega_{j}^{2}+i \omega \omega_{j} / Q_{j}}$,

where

$A_{j}(\omega)=\frac{\alpha(\gamma-1)}{V_{c}} \int p_{j}^{*} \cdot I \mathrm{~d} V$,

and

$f_{l m n}=\frac{c}{2} \sqrt{\left(\frac{l}{L}\right)^{2}+\left(\frac{\alpha_{m n}}{r_{C}}\right)^{2}}$,

where $\alpha_{m n}$ is the zeros of the derivative of the Bessel functions, $L$ is the length of the PA cell, $r_{c}$ is the radius of the PA cell, $l$ is the longitudinal quantum number, $m$ is the azimuthal quantum number, and $n$ is the radial quantum number. ${ }^{49,50}$

\subsection{Photoacoustic Cell Simulations}

The acoustic properties of the PA chamber were studied using finite element software from COMSOL (Burlington, Massachusetts). The pressure acoustics, frequency domain (acpr) user interface was used for the present study. The governing equations in the pressure acoustic model were formulated using the total pressure in a scattered field formulation. The solution gives the acoustic variation/excess pressure $(p)$ to the ambient pressure. The solved Helmholtz wave equations are ${ }^{51}$

$\nabla \cdot \frac{1}{\rho_{c}}\left(\nabla \rho_{t}-q_{d}\right)-\frac{k_{\mathrm{eq}}^{2} \rho_{t}}{\rho_{c}}=Q_{m}$,

where

$\rho_{t}=\rho_{s}+\rho_{b}$,

$k_{\mathrm{eq}}^{2}=\left(\frac{\omega}{c_{c}}\right)^{2}$,

$c_{c}=c$ and $\rho_{c}=\rho$,

where $c$ is the speed of sound, $\rho$ is the density of material, $t$ is the time, $\rho_{t}$ is the total acoustic pressure, $\rho_{s}$ is the scattered pressure field, $\rho_{b}$ is the background pressure field, $k_{\mathrm{eq}}$ is the amplitude of the wave vector $k, \omega$ is the angular frequency, and $q_{d}$ and $Q_{m}$ are acoustic dipole and monopole source terms, respectively. The subscript $c$ to the density $\rho$ and the speed of sound $c$ represent the complex speed of sound and density, respectively. ${ }^{36}$ Lossy media such as porous materials, highly viscous fluids, etc., are modeled by using the complex-valued speed of sound $\left(c_{c}\right)$ and density $\left(\rho_{c}\right)$. Since air is an acoustic transport medium, $c_{c}$ and $\rho_{c}$ in our case become $c$ and $\rho$, respectively.

The purpose of this study is to optimize the PA cell for maximum output. The generation of the PA signal amplitude is inversely proportional to the cell volume. Larger cell volumes result in weaker signals due to the damping within the cell. Conversely, for small cell volumes, the thermal diffusion length and viscous-thermal damping distance of air progressively become dominant. The thermal diffusion length and viscousthermal damping distance of air were also taken into account in designing the cell. The thermal diffusion length and viscous-thermal damping of gas are dependent on frequency, temperature, and pressure. At room temperature, pressure, and at a frequency of $100 \mathrm{~Hz}$, the air has a thermal diffusion length and viscous-thermal damping distance of 0.2 and $0.1 \mathrm{~mm}$, respectively. ${ }^{11}$ A PA sensor to work in a broad range of modulation frequencies should need minimum dimensions of 1 to $2 \mathrm{~mm}$ to avoid any PA signal saturation due to acoustic damping of PA cell walls. ${ }^{11}$ The influence of this effect on the PA signal was investigated by adding thermoacoustic physics to the pressure acoustic model.

\subsubsection{Study of the effect of sample chamber on PA signal}

First, the effect of Sc size on the PA signal was investigated. The diameters of the Sc chosen for the studies varied between 2 and $8 \mathrm{~mm}$. The height of the chamber was $3 \mathrm{~mm}$. The height was chosen to $3 \mathrm{~mm}$ in order to leave enough room to accommodate the sample, and $1 \mathrm{~mm}$ resonator though $2 \mathrm{~mm}$ enough to avoid the thermo-viscous loses caused by cell surface. The length of the resonator column used in the study was $100 \mathrm{~mm}$. 
The PA source is a sample heated by a laser, which is represented by a heat source $(\mathrm{Q})$ in the simulation. The heat source was simulated to exhibit a Gaussian heat distribution (a TEM 00 mode laser was used for the ORPAM). The Gaussian heat distribution is given as

$Q_{0}=Q \exp \left(\frac{-r^{2}}{2 \sigma_{0}^{2}}\right)$

where $\sigma_{0}$ is the laser beam Gaussian spread function and $Q$ is the heat input amplitude. Since the laser beam was focused using $10 \times, 0.25 \mathrm{NA}$ objective, the irradiance produced was $1 \mathrm{~W} / \mathrm{mm}^{2}$. In experiments, carbon black was used. Carbon black has a heat conversion efficiency from 0.5 to 1 depending on the material. Assuming the light to the heat conversion efficiency of the sample to be 0.5 , the heat produced by $1 \mathrm{~W} / \mathrm{mm}^{2}$ irradiance would be $1 \mathrm{~J} / \mathrm{mm}^{2}$.

When the sound waves propagate in a fluid bounded by walls, viscous and thermal boundary layers will be created close to the PA cell surfaces due to the interaction of the fluid with solid PA cell wall and the difference in the thermal conductivities of the liquid and the solid wall. In the case of a small PA chamber viscous and thermal losses can be significant. A no-slip $(u=0)$ and isothermal $[-\vec{n} \cdot(-k \cdot \nabla T)=0]$ conditions were employed to all the boundaries of the simulation region. Nodal constrain boundary condition was chosen in order to account any attenuation caused by sharp edges of the PA chamber.

Incorporating the microphone and lock-in response in the numerical simulation. The frequency response of the PA sensor is the convolution of the response of the microphone and a lock-in amplifier. The microphone response at low frequencies is not flat. The microphone frequency response can be described by simpler acoustic diaphragm impedance models. ${ }^{52}$ Figure 3 shows the equivalent circuit of the acoustic diaphragm impedance of the microphone. The components represent stiffnesses, masses, and damping of the electromechanical system. The total impedance of the circuit is

$z=\frac{1}{i \omega c_{a c}}+R_{a c}+i \omega L_{a c}$

where $C_{a c}$ is the diaphragm system compliance, $L_{a c}$ is the diaphragm system mass, and $R_{a c}$ is the diaphragm system resistance.

The effect of the microphone is accounted for in the simulation by applying serial coupling RCL impedance boundary condition with values of $C_{\mathrm{ac}}, L_{\mathrm{ac}}$, and $R_{\mathrm{ac}}$ at the microphone end (Table 1).

The microphone response $L_{0}$ (for a constant volume displacement source): ${ }^{53}$

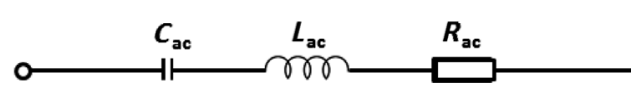

Acoustic terminals

-

Fig. 3 A simplified model representing the acoustical impedance of the microphone.

Table 1 Values of the simplified microphone model shown in Fig. 3.

Acoustic parameter

\begin{tabular}{lc}
\hline$R_{\mathrm{ac}}$ & $2.83 \times 10^{-13} \mathrm{Ns} / \mathrm{m}^{5}$ \\
$C_{\mathrm{ac}}$ & $896 \mathrm{~m}^{5} / \mathrm{N}$ \\
$L_{\mathrm{ac}}$ & $56.2 \times 10^{6} \mathrm{~kg} / \mathrm{m}^{4}$ \\
\hline
\end{tabular}

$L_{0}=10 \log \left(\frac{\left\langle P_{\mathrm{mic}}\right\rangle^{2}}{P_{\mathrm{ref}}^{2}}\right)$

where $\left\langle P_{\text {mic }}\right\rangle^{2}$ is the root mean square pressure at the measurement microphone and $P_{\text {ref }}$ is the reference pressure.

Since the output of the microphone is preamplified by the amplifier in the microphone, the frequency response of the system will be the combination of both the electrical response of the preamplifier and the acousto-mechanical response of the microphone. ${ }^{52}$ The electrical circuit of the combined system that comprising of both the microphone and the preamplifier is shown in Fig. 4.

The electrical frequency response of the circuit is given as

$$
\frac{V_{0}}{V_{0 c}}=\frac{C_{m}}{C_{m}+C_{i}} \cdot \frac{j \omega\left(C_{m}+C_{i}\right) R_{i}}{1+j \omega\left(C_{m}+C_{i}\right) R_{i}} \cdot g \cdot \frac{1}{1+j C_{c} R_{0}},
$$

where $V_{0 c}$ is the open circuit microphone voltage, $V_{0}$ is the output voltage, $C_{m}$ is the microphone capacitance, $C_{i}$ is the preamplifier input capacitance, $R_{i}$ is the preamplifier input resistance, $g$ is the amplifier gain, $R_{0}$ is the preamplifier output resistance, and $C_{c}$ is the cable capacitance whose typical values are given in Table 2.

The output of the PSD of the lock-in amplifier contains many signals. The main components are the sum and difference frequency of the input and the reference signals. The low pass filter at the PSD output filters out all the unwanted AC signals that include $2 F$ (sum frequency) and the noise components but records the dc component which is the input signal detected by a lock-in at the reference frequency. As the measurement was made using the lock-in amplifier set at a first-order low pass filter configuration with a time constant $(\tau)$, the transfer function of the lock-in amplifier, in this case, is $T(\omega)=\left|\frac{V_{o}}{V_{i}}\right|=\frac{1}{\sqrt{1+(\omega \tau)^{2}}}$, where $V_{o}$ is the output, $V_{i}$ is the input, and $\omega$ is the angular

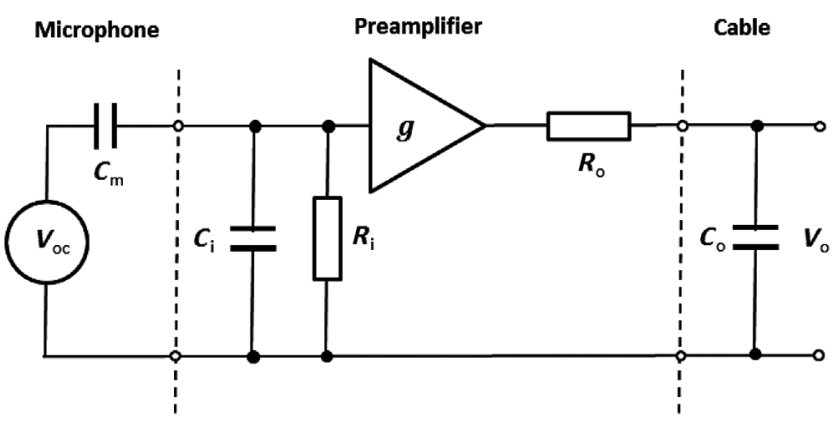

Fig. 4 An simple model for calculation of the electrical frequency response of a microphone and its preamplifier loaded with a cable. 
Table 2 Values of electrical circuit elements of the simplified model shown in Fig. 4.

\begin{tabular}{lc} 
Symbol & Typical value \\
\hline$V_{0 c}$ & $1 \mu \mathrm{V}$ to $50 \mathrm{~V}$ \\
$C_{m}$ & $18 \mathrm{pF}$ \\
$C_{i}$ & $0.2 \mathrm{pF}$ \\
$R_{i}$ & $10 \mathrm{G} \Omega$ \\
$g$ & 0.995 \\
$R_{0}$ & $30 \Omega$ \\
$C_{c}$ & $3 \mathrm{nF}$ \\
\hline
\end{tabular}

frequency. Hence, the signals of frequencies less than $1 / \tau$ were passed but frequencies $\omega \tau \gg 1$ were attenuated by $T \rightarrow 1 / \omega \tau$. The transfer function of the lock-in in this configuration was measured experimentally using a nanosecond response PD from Thorlabs (please refer to Fig. S3 in the Supplemental Materials). The electrical frequency response of the microphone [Eq. (13)] and the experimentally measured transfer function (Fig. S3 in the Supplemental Materials) of the lock-in amplifier were multiplied with the result of the numerical simulation to generate the PA response [Eq. (12)] of the cell. Finally, the PA response was squared according to Eq. (3) as the PSD of lock-in involved the multiplication of the input and the reference signal.

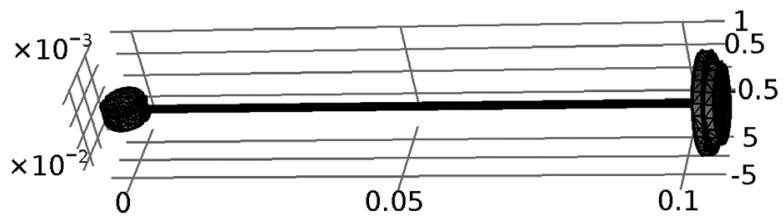

Fig. 5 The meshing of the PA cell of chamber size $8 \mathrm{~mm} \times 3 \mathrm{~mm}$ with a resonator of length $100 \mathrm{~mm}$.

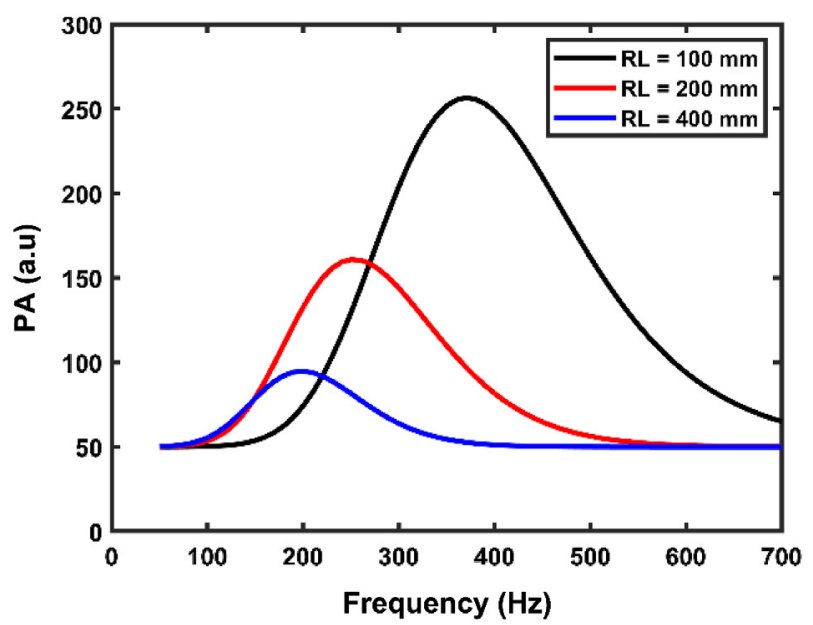

(a)

\subsubsection{Study of the effect of resonance tunnel on $P A$ signal}

The resonance of the PA cell can be tuned by changing the length of the resonator column/Helmholtz resonator. The narrow cylindrical channel connecting Sc and microphone acts as the Helmholtz resonator. The Sc end of the resonator acts as an open-end while the microphone end acts as a close end. The resonator of lengths 100, 200, and $400 \mathrm{~mm}$ was investigated. The air was the acoustic transport medium. The resonator of diameter $1 \mathrm{~mm}$ is chosen for the study. The entire geometry was meshed using the extremely fine precondition in COMSOL. Figure 5 represents the $8 \mathrm{~mm} \times 3 \mathrm{~mm}$ PA sensor with a resonator of length $100 \mathrm{~mm}$ used in COMSOL. The sensor is meshed by extremely fine precondition for numerical simulation.

The configuration was solved for the pressure distribution with frequency ranging from 50 to $1000 \mathrm{~Hz}$.

\section{Results and Discussion}

Figure 6 shows the simulated and measured PA signals as a function of frequency for resonator lengths 100, 200, and $400 \mathrm{~mm}$. The resonance peak was decreased with an increase in resonator column length, which is expected, as the frequency is inversely proportional to the resonator length [Eq. (8)]. The experimental results exhibited a similar trend to the numerical simulations. Table 3 compares the resonance frequencies of the

Table 3 Comparison of resonance frequencies obtained from experimental measurements and numerical simulations for various resonator lengths.

\begin{tabular}{lccc}
$\begin{array}{l}\text { Sample chamber } \\
\text { size } 8 \mathrm{~mm} \times 3 \mathrm{~mm}\end{array}$ & $\begin{array}{c}\text { Frequency }(\mathrm{Hz}) \\
\text { (numerical) }\end{array}$ & $\begin{array}{c}\text { Frequency }(\mathrm{Hz}) \\
\text { (experiment) }\end{array}$ & $\begin{array}{c}\text { Percentage } \\
\text { difference }\end{array}$ \\
\hline $\begin{array}{l}\text { Resonator length } \\
R_{I}=100 \mathrm{~mm}\end{array}$ & 370 & 377 & 1.87 \\
$R_{I}=200 \mathrm{~mm}$ & 240 & 231 & 3.82 \\
$R_{I}=400 \mathrm{~mm}$ & 200 & 174 & 13.9 \\
\hline
\end{tabular}

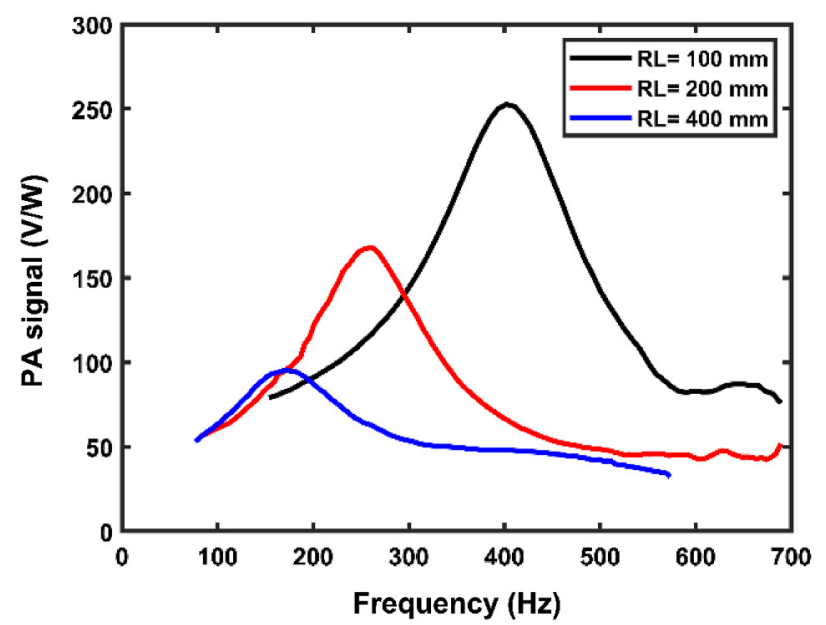

(b)

Fig. 6 The frequency response of the PA sensor for various resonator lengths from (a) theory and (b) experiment. 


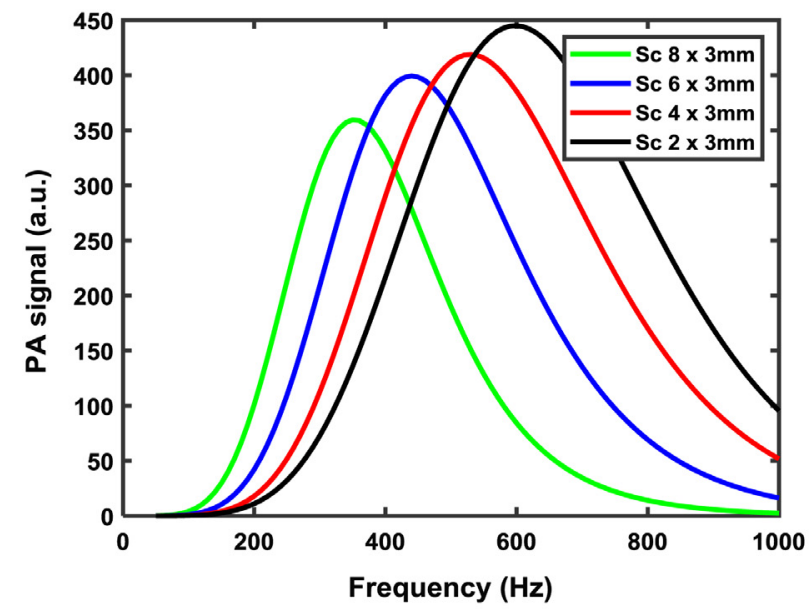

(a)

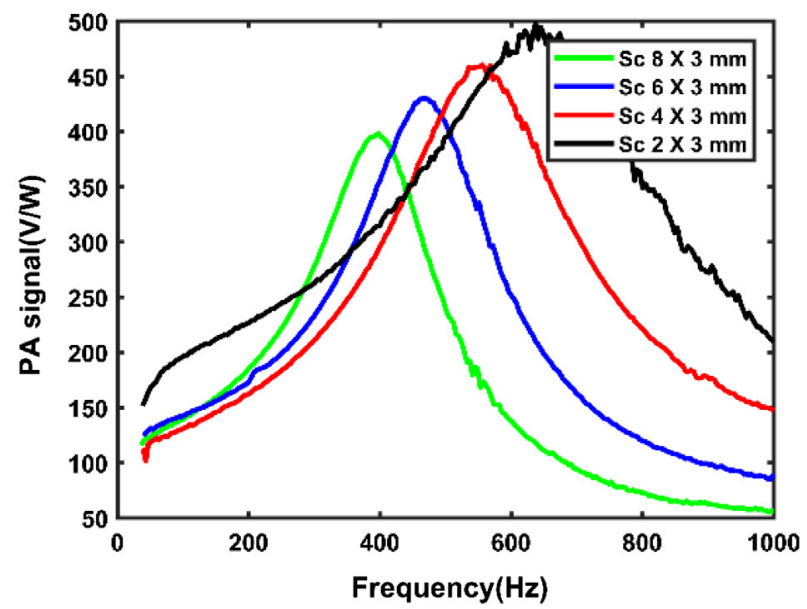

(b)

Fig. 7 The frequency response of the PA sensor for sample chambers of various sizes (a) simulation and (b) experiment.

sensor with various resonator lengths obtained from both numerical simulations and experimental measurements.

Next, we investigated the effect of the sample chamber size on the resonance frequency of the PA sensor. The sample chamber sizes investigated were $8 \mathrm{~mm} \times 3 \mathrm{~mm}, 6 \mathrm{~mm} \times 3 \mathrm{~mm}$, $4 \mathrm{~mm} \times 3 \mathrm{~mm}$, and $2 \mathrm{~mm} \times 3 \mathrm{~mm}$. The resonator column length in all cases was $R_{L}=100 \mathrm{~mm}$. Figure 7(a) shows the measured resonance spectra normalized to the incident power.

The resonance frequency of the chamber was increased with decreasing sample chamber size. The increment was due to the combined effect of the sample chamber and resonator column. The combination acts as a single resonator column with a formation of the acoustic node at the microphone end and antinode at the sample chamber end. Figure 7(b) shows the numerical simulation results, and Table 4 shows the comparison of the numerical and experimental values.

The PA signal amplitude also increased with decreasing sample chamber size. The PA amplitude was $43.4 \mathrm{~V} / \mathrm{W}$ larger in the case of $2 \mathrm{~mm} \times 3 \mathrm{~mm}$ size compared to the $8 \mathrm{~mm} \times 3 \mathrm{~mm}$ size. This is due to damping caused by the air column in the cell. The PA signal is inversely proportional to the cell volume. However, the cell of smaller size would create acoustic damping losses caused by cell boundaries, which would be observed as a broadening of the resonance peak.

Table 4 Comparison of resonance frequencies obtained from the experimental and numerical simulation for the various sample chamber sizes, using a fixed resonator length of $100 \mathrm{~mm}$.

\begin{tabular}{lccc}
\hline $\begin{array}{l}\text { Sample } \\
\text { chamber size }\end{array}$ & $\begin{array}{c}\text { Resonant } \\
\text { frequency }(\mathrm{Hz}) \\
\text { simulation }\end{array}$ & $\begin{array}{c}\text { Resonant } \\
\text { frequency }(\mathrm{Hz}) \\
\text { experiment }\end{array}$ & $\begin{array}{c}\text { Percentage } \\
\text { difference }\end{array}$ \\
\hline $8 \mathrm{~mm} \times 3 \mathrm{~mm}$ & 370 & 377 & 1.87 \\
$6 \mathrm{~mm} \times 3 \mathrm{~mm}$ & 480 & 453 & 5.78 \\
$4 \mathrm{~mm} \times 3 \mathrm{~mm}$ & 540 & 536 & 0.74 \\
$2 \mathrm{~mm} \times 3 \mathrm{~mm}$ & 610 & 613 & 0.49 \\
\hline
\end{tabular}

The quality factor $\left(Q_{j}\right)$ of the system was estimated from the broadening of the peak.

Table 5 shows the quality factor $Q_{j}$ of the PA sensor for various chamber sizes which was obtained by fitting Eq. (6) to the resonance frequency response of the various PA sensors. The larger sample chamber exhibited a better-quality factor than the smaller ones. For example, the Sc of size $8 \mathrm{~mm} \times 3 \mathrm{~mm}$ had a $37 \%$ greater quality factor than the $2 \mathrm{~mm} \times 3 \mathrm{~mm} \mathrm{Sc}$.

We have investigated numerically the possible eigenmodes supported by the PA cell. The analytical expression 8 used to identify the type of eigenmodes excited. Table 6 reports the

Table 5 Quality factor of the PA sensor with various sample chamber sizes.

\begin{tabular}{lc}
\hline Sample chamber & $\begin{array}{c}\text { Quality factor } \\
\text { (Experiment) }\end{array}$ \\
\hline $8 \mathrm{~mm} \times 3 \mathrm{~mm}$ & 2.52 \\
$6 \mathrm{~mm} \times 3 \mathrm{~mm}$ & 2.49 \\
$4 \mathrm{~mm} \times 3 \mathrm{~mm}$ & 2.49 \\
$2 \mathrm{~mm} \times 3 \mathrm{~mm}$ & 1.84 \\
\hline
\end{tabular}

Table 6 Analytical results of eigenmodes supported by the resonator.

\begin{tabular}{lcccc}
$\begin{array}{l}\text { Resonator } \\
\text { length }(\mathrm{mm})\end{array}$ & $\begin{array}{c}\text { Mode 100 } \\
\text { frequency } \\
(\mathrm{Hz})\end{array}$ & $\begin{array}{c}\text { Mode 010 } \\
\text { frequency } \\
(\mathrm{Hz})\end{array}$ & $\begin{array}{c}\text { Mode 001 } \\
\text { frequency } \\
(\mathrm{Hz})\end{array}$ & Type \\
\hline Sc $(8 \mathrm{~mm} \times 3 \mathrm{~mm})$ & 1657 & $194.2 \times 10^{3}$ & $404 \times 10^{3}$ & $\lambda / 4$ \\
\hline 100 & 829 & $194.2 \times 10^{3}$ & $404 \times 10^{3}$ & $\lambda / 4$ \\
200 & 414 & $194.2 \times 10^{3}$ & $404 \times 10^{3}$ & $\lambda / 2$ \\
400 & & & & \\
\hline
\end{tabular}


Table 7 Comparison of experimental, numerical, and analytical results of eigenmodes supported by the resonator.

\begin{tabular}{lccc}
$\begin{array}{l}\text { Resonator } \\
\text { length }(\mathrm{mm})\end{array}$ & $\begin{array}{c}\text { Resonant } \\
\text { frequency }(\mathrm{Hz}) \\
\text { experiment }\end{array}$ & $\begin{array}{c}\text { Resonant } \\
\text { frequency }(\mathrm{Hz}) \\
\text { numerical }\end{array}$ & $\begin{array}{c}\text { Resonant } \\
\text { frequency }(\mathrm{Hz}) \\
\text { analytical }\end{array}$ \\
\hline Sc $(8 \mathrm{~mm} \times 3 \mathrm{~mm})$ & 377 & 370 & $414 \mathrm{~Hz}$ \\
\hline 100 & 231 & 240 & 207 \\
200 & $174 \mathrm{~Hz}$ & $200 \mathrm{~Hz}$ & 203 \\
$400 \mathrm{~mm}$ & & & \\
\hline
\end{tabular}

frequencies of the first three lower-order eigenmodes supported by the PA sensor. Only the mode (100) was able to observe in this case due to the modulation range exhibited by the chopper. The mode (100) represents the longitudinal acoustic mode. In our case, we observed the longitudinal mode of type $\lambda / 4$ with the open end in the sample chamber and close end at the microphone end. Table 7 represents the comparison of experimental and analytical results. The difference compared to the analytical results was due to that in Eq. (5), we have not accounted for the resonance tube end correction in the expression. ${ }^{54}$

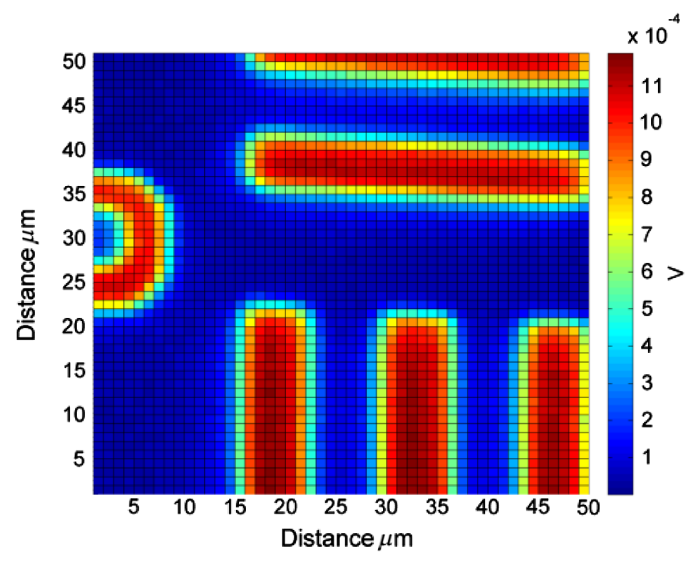

(a)
Next, the resolution and molecular sensitivity of the system were calculated. The PA cell of dimensions $(\mathrm{Sc}=8 \mathrm{~mm} \times$ $3 \mathrm{~mm}$ and $\mathrm{Rl}=100 \mathrm{~mm}$ ) was chosen for the study. A USAF 1951 resolution chart and RBC were used for resolution and molecular sensitivity measurements.

Figures $8(\mathrm{~b})$ and $8(\mathrm{c})$ show the PA image of the group 7 element 6 and the plot of line spread function (LSF) of one of the components of the element. The lateral resolution of a system defined as its ability to distinguish the two nearest-neighboring objects clearly. This can be estimated experimentally by quantifying the edge blurring or edge spread function of the image. The region in the solid rectangular box in the PA image used to calculate the edge spread function (EDF). The EDF is a step function converted to a Gaussian profile to find the edge spread with the help of Gaussian fit. The EDF converted to Gaussian profile by differentiating the data. The differentiated EDF is called the LSF. The star marker in the figure represents the LSF. The Gaussian fit (solid red line) on the LSF gives a lateral resolution of $1.9 \mu \mathrm{m}$.

The molecular-sensitivity measurement of the system is also called as noise equivalent sensitivity because it depends on the total noise of the system. It provides information about the minimum number of molecules required to generate a PA signal greater than the system noise. RBCs were used to measure the molecular sensitivity of the system because the RBC is the

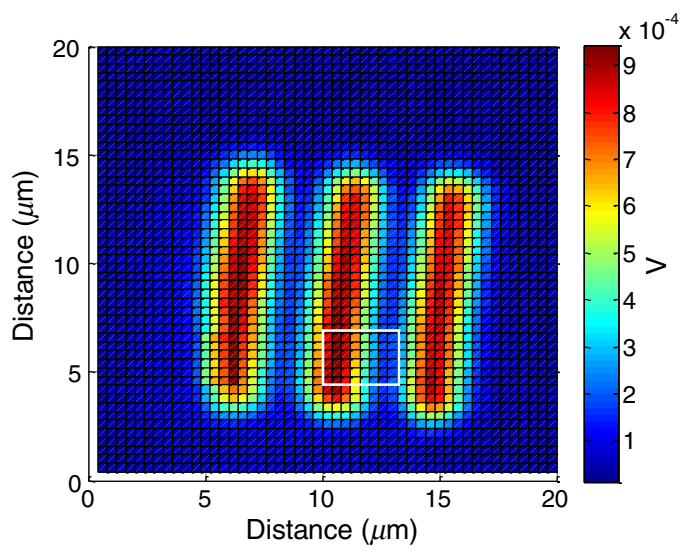

(b)

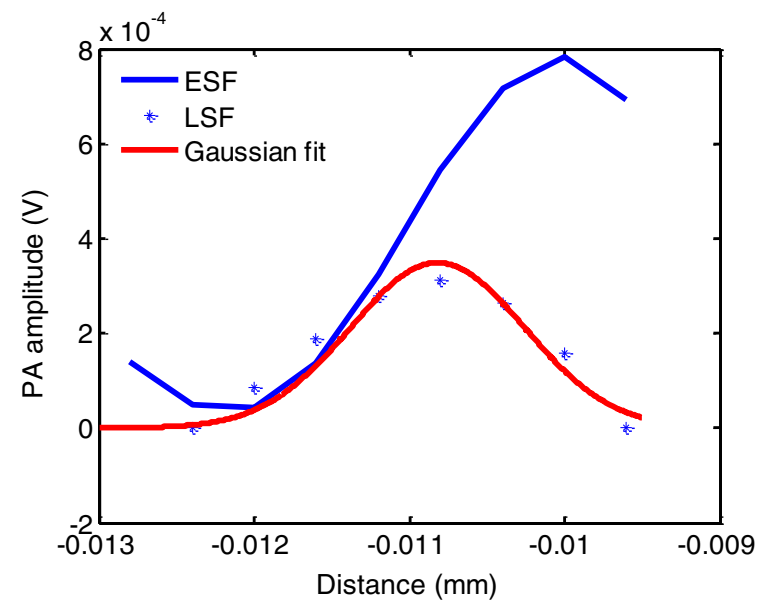

(c)

Fig. 8 PA image of a lateral resolution chart, USAF 1951 (a) group 6 element 6, (b) group 7 element 6 , and (c) LSF, where the solid blue line is the ESF, marker * is the LSF, and the solid red line is Gaussian fit. 


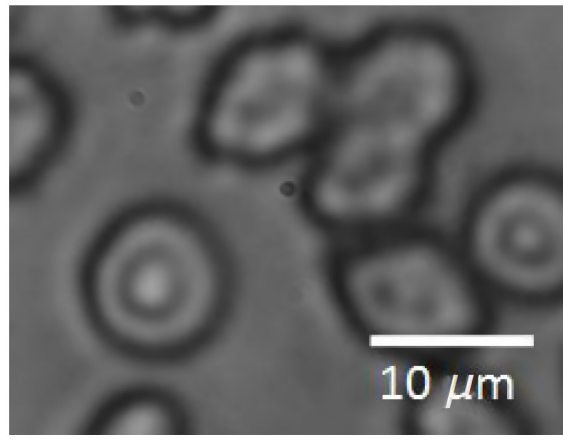

(a)

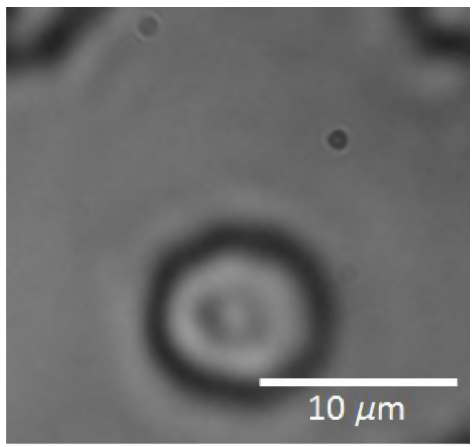

(c)

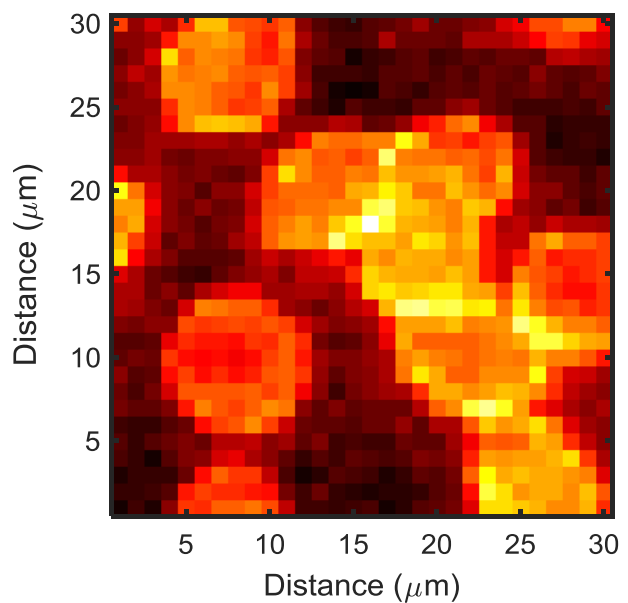

(b)

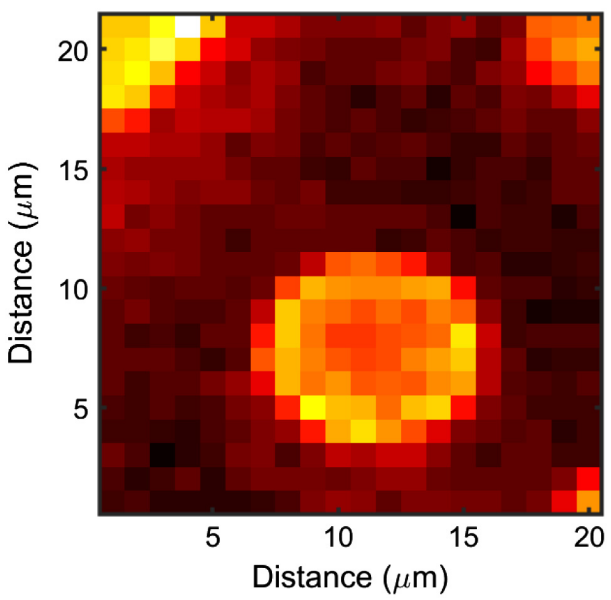

(d)

Fig. 9 (a) Optical and (b) PA image of blood smear on a microscope glass slide measured by transillumination method. (c) Optical and (d) PA image measured by the reflection method.

dominant endogenous chromophore in most PA imaging as it exhibits a very strong absorption cross-section in the optical and near-infrared wavelength.

Measurements performed on a common blood smear on a glass slide to probe single RBC. Figure 9 shows the optical and PA images of the RBCs. The measurement was also made in reflection mode [Fig. 9(b)] as conditions are relevant to in vivo measurements. Figure $9(\mathrm{~d})$ shows the PA image measured in the reflection mode.

\section{Discussion}

Table 3 compares the numerical simulation and experiment results of PA resonance of various resonator lengths. The numerical simulation and experiment results show good agreement. The small difference in peak value between the experimental and simulation results could be due to the variation in mesh spacing between PA sensor of various sizes. The variation is bigger in the case of $\mathrm{Rl}=400 \mathrm{~mm}$ probably due to acoustic attenuation losses increase with traveling distance.

The amplitude of the resonance peak (Fig. 6) decreased with increasing in resonator length. The value decreased from
$104.8 \mathrm{~V} / \mathrm{W}$ for the resonator of length $400 \mathrm{~mm}$ to $43.86 \mathrm{~V} / \mathrm{W}$ for the resonator of length $100 \mathrm{~mm}$. The decrease in the PA amplitude could be due to attenuation of the acoustic signal by the thermo-viscous damping along the long resonator column.

We have investigated the attenuation loss due to PA cell chamber size. The PA cell of size $8 \mathrm{~mm} \times 3 \mathrm{~mm}$ (Table 5) exhibited a better quality factor compared to the smaller size. Thermal loss and acoustic damping can play a significant role in a smaller PA sample chamber. For example, air at room temperature and pressure has a thermal diffusion length of about $2 \mathrm{~mm}$ for a modulation frequency of $100 \mathrm{~Hz}$, whereas thermo-viscous damping is $0.1 \mathrm{~mm}$. Therefore, the sample chamber below the size of $2 \mathrm{~mm}$ should be avoided to study a material that has strong optical absorption and emissivity because PA signal saturation could be observed. However, a smaller PA chamber could be preferred to study samples of weaker optical absorption as it provides a stronger PA signal.

The PA cell also suffers from other losses such as volume loss and gas imperfection loss. The volume loss is caused by the friction of neighboring gas layers due to thermal conduction and viscosity in the gas volume. ${ }^{48,49}$ 
Table 8 A summary of the sensor performance parameters.

\begin{tabular}{lcc} 
Sensor property & Old sensor & New sensor \\
\hline NEM & $693 \times 10^{2}$ & $316 \times 10^{2}$ \\
$\begin{array}{l}\text { NEM per unit } \\
\text { bandwidth }\end{array}$ & $43,887 \mathrm{~Hz}^{-1 / 2}$ or & $19,966 \mathrm{~Hz}^{-1 / 2}$ or \\
MPDL & 72.8 zeptomoles & 33 zeptomoles \\
MPDL per unit & $19.1 \mu \mathrm{Pa}$ & $8.7 \mu \mathrm{Pa}$ \\
bandwidth & $7.64 \mu \mathrm{Pa} / \mathrm{Hz}^{1 / 2}$ & $3.5 \mu \mathrm{Pa} / \mathrm{Hz}^{1 / 2}$ \\
\hline
\end{tabular}

Finally, the resolution and the molecular sensitivity of the system were investigated. A USAF chart and RBCs were used for these purposes. The lateral resolution of the system was $1.9 \mu \mathrm{m}$. The SNR of the system calculated by estimating the average PA signal with and without the RBC (Fig. 9). The point of interrogation was $1.2 \mu \mathrm{m}$ as a $40 \times$ objective was used for imaging. The average PA signal from the RBC and the background were $2.12 \times 10^{-4} \mathrm{~V}$ and $9.54 \times 10^{-7} \mathrm{~V}$, respectively. The SNR of the system was $\sim 220$.

Based on these measurements, the molecular sensitivity/ molecular concentration equivalent to SNR was calculated. A single RBC contains about $270 \times 10^{6}$ hemoglobin molecules. ${ }^{55}$ The mean corpuscular volume is $92.8 \times 10^{-15} \mathrm{~L}$. The estimated concentration of hemoglobin in a single RBC is $3.1 \times 10^{6} \mu \mathrm{m}^{-3}$. A $40 \times$ objective of 0.6 NA with an input light beam of a diameter of $0.36 \mathrm{~mm}$ excites about $\sim 7 \times 10^{6}$ hemoglobin molecules. The average PA signal detected was $2.12 \times 10^{-4} \mathrm{~V}$. The measured system molecular-sensitivity equivalent to noise [or noise equivalent sensitivity $(\mathrm{NEM})]$ was $\sim 316 \times 10^{2}$. As the bandwidth of the lock-in amplifier filter was $1.5 \mathrm{~Hz}$, the NEM per unit bandwidth $(\mathrm{NEM} / \sqrt{ } \Delta f)$ was $\sim 19,966 \mathrm{~Hz}^{-1 / 2}$ or $33 \times$ $10^{-21} \mathrm{~mol}$ or 33 zeptomoles. Table 8 summaries the sensitivity and minimum detectable pressure of the system. The system exhibited sensitivity about 2.2 times more than our previously reported system. ${ }^{38}$

Finally, the minimum pressure measurable by the system was calculated. Our microphone sensitivity was $50 \mathrm{mV} / \mathrm{Pa}$. The PA signal was measured with zero amplifier gain. Therefore, the measured PA signal of the sensor is proportional to the pressure, i.e., $50 \mathrm{mV} / \mathrm{Pa}$. The minimum measurable pressure is the pressure equivalent to NEM sensitivity of the system. The MPDL of the PA sensor was $8.7 \mu \mathrm{Pa}$. The MPDL per unit bandwidth, MPDL $/ \sqrt{ } \Delta f=3.4 \mu \mathrm{Pa} / \mathrm{Hz}^{1 / 2}$.

\section{Conclusions}

This study presents numerical simulations and experimental validation of a recently developed noncontact PA sensor for lowpower CW laser-based frequency-domain PA microscopy. The main components of the PA sensor are the sample chamber and resonator column, which were optimized to maximize the signal. Various sample chamber and resonator column sizes were investigated. It was found that the resonance frequency of the PA cell decreased with an increase in the resonator length and that the resonance frequency increased with a decrease in the sample chamber size. The $8 \mathrm{~mm} \times 3 \mathrm{~mm}$ sample chamber exhibited a better quality factor compared to the other sizes examined (about 27\% higher than the $2 \mathrm{~mm} \times 3 \mathrm{~mm} \mathrm{Sc}$ ). We attribute the drop in quality factor for the smaller cell to viscous-thermal damping at the cell boundaries. Therefore, an Sc of minimum size $2 \mathrm{~mm}$ is suitable for PA imaging. The system exhibited NEM sensitivity per unit frequency was $\sim 19,966 \mathrm{~Hz}^{-1 / 2}$ or $33 \times 10^{-21} \mathrm{~mol}$ or 33 zeptomol and an MPDL per unit bandwidth, MPDL $/ \sqrt{ } \Delta f=3.4 \mu \mathrm{Pa} / \mathrm{Hz}^{1 / 2}$. The developed $\mathrm{PA}$ sensor could be an inexpensive add-on component to existing commercial microscopes, which could use low-power CW or even pulse xenon lamp or LED sources. The developed PA sensor is cost effective and expected to have many applications, such as cellular imaging, surface tissue imaging, and sensing, and interrogating optically opaque samples and highly scattering sample such as powders.

\section{Disclosures}

The authors have no relevant financial interests in this article and no potential conflicts of interest to disclose.

\section{Acknowledgments}

This work was funded by the Natural Sciences and Engineering Research Council of Canada (RGPIN-2017-06496) and Ryerson University. Funding to purchase the equipment was provided by the Canada Foundation for Innovation, the Ontario Ministry of Research and Innovation, and Ryerson University.

\section{References}

1. A. G. Bell, "On the production and reproduction of sound by light," Am. J. Sci. s3-20(118), 305-324 (1880).

2. A. Rosencwaig, Photoacoustics and Photoacoustic Spectroscopy, John Wiley \& Sons Inc., New York (1980).

3. E. L. Kerr and J. G. Atwood, "The laser illuminated absorptivity spectrophone: a method for measurement of weak absorptivity in gases at laser wavelengths," Appl. Opt. 7(5), 915-921 (1968).

4. L. B. Kreuzer, "Ultralow gas concentration infrared absorption spectroscopy," J. Appl. Phys. 42(7), 2934-2943 (1971).

5. L. B. Kreuzer, N. D. Kenyon, and C. K. N. Patel, "Air pollution: sensitive detection of ten pollutant gases by carbon monoxide and carbon dioxide lasers," Science 177(4046), 347-349 (1972).

6. F. Müller et al., "Transportable, highly sensitive photoacoustic spectrometer based on a continuous-wave dualcavity optical parametric oscillator," Opt. Express 11(22), 2820-2825 (2003).

7. A. Danielli et al., "Label-free photoacoustic nanoscopy," J. Biomed. Opt. 19(8), 086006 (2014).

8. M. Xu and L. V. Wang, "Photoacoustic imaging in biomedicine," Rev. Sci. Instrum. 77(4), 041101 (2006).

9. B. Cox et al., "Quantitative spectroscopic photoacoustic imaging: a review," J. Biomed. Opt. 17(6), 061202 (2012).

10. P. Beard, "Biomedical photoacoustic imaging," Interface Focus 1(4), 602-631 (2011).

11. A. Rosencwaig, "Photoacoustic spectroscopy of solids," Opt. Commun. 7(4), 305-308 (1973).

12. J. B. Kinney and R. H. Staley, "Applications of photoacoustic spectrosсору," Апnи. Rev. Mater. Sci. 12(1), 295-321 (1982).

13. A. Rosencwaig, "Photoacoustic spectroscopy," Annu. Rev. Biophys. Bioeng. 9(1), 31-54 (1980).

14. H. F. Zhang et al., "Functional photoacoustic microscopy for highresolution and noninvasive in vivo imaging," Nat. Biotechnol. 24(7), 848-851 (2006).

15. J. Yao et al., "High-speed label-free functional photoacoustic microscopy of mouse brain in action," Nat. Methods 12(5), 407-410 (2015).

16. E. M. Strohm, E. S. L. Berndl, and M. C. Kolios, "High frequency labelfree photoacoustic microscopy of single cells," Photoacoustics 1(3-4), 49-53 (2013).

17. J. Yao and L. V. Wang, "Photoacoustic microscopy," Laser Photonics Rev. 7(5), 758-778 (2013). 
18. Y. Zhang et al., "Non-invasive multimodal functional imaging of the intestine with frozen micellar naphthalocyanines," Nat. Nano 9(8), 631-638 (2014).

19. E. M. Strohm, M. J. Moore, and M. C. Kolios, "Single cell photoacoustic microscopy: a review," IEEE J. Sel. Top. Quantum Electron. 22(3), 137-151 (2016)

20. L. Zhu et al., "Multiview optical resolution photoacoustic microscopy," Optica 1(4), 217-222 (2014).

21. S. Park et al., "Acoustic resolution photoacoustic microscopy," Biomed. Eng. Lett. 4(3), 213-222 (2014).

22. M. J. Moore et al., "Photoacoustic F-mode imaging for scale specific contrast in biological systems," Coтmun. Phys. 2(1), 30 (2019).

23. E. Hysi et al., "Insights into photoacoustic speckle and applications in tumor characterization," Photoacoustics 14, 37-48 (2019).

24. S. A. Carp and V. Venugopalan, "Optoacoustic imaging based on the interferometric measurement of surface displacement," J. Biomed. Opt. 12(6), 064001 (2007).

25. A. Hochreiner et al., "Non-contact photoacoustic imaging using a fiber based interferometer with optical amplification," Biomed. Opt. Express 4(11), 2322-2331 (2013).

26. Y. Wang, C. Li, and R. K. Wang, "Noncontact photoacoustic imaging achieved by using a low-coherence interferometer as the acoustic detector," Opt. Lett. 36(20), 3975-3977 (2011).

27. J. Eom, S. J. Park, and B. H. Lee, "Noncontact photoacoustic tomography of in vivo chicken chorioallantoic membrane based on all-fiber heterodyne interferometry," J. Biomed. Opt. 20(10), 106007 (2015).

28. E. Zhang, J. Laufer, and P. Beard, "Backward-mode multiwavelength photoacoustic scanner using a planar Fabry-Perot polymer film ultrasound sensor for high-resolution three-dimensional imaging of biological tissues," Appl. Opt. 47(4), 561-577 (2008).

29. B. P. Payne et al., "Optoacoustic tomography using time-resolved interferometric detection of surface displacement," J. Biomed. Opt. 8(2), 273-280 (2003).

30. P. C. Beard, F. Perennes, and T. N. Mills, "Transduction mechanisms of the Fabry-Perot polymer film sensing concept for wideband ultrasound detection," IEEE Trans. Ultrason. Ferroelectr. Freq. Control 46(6), 1575-1582 (1999).

31. R. A. Barnes et al., "Probe beam deflection technique as acoustic emission directionality sensor with photoacoustic emission source," Appl. Opt. 53(3), 511-519 (2014).

32. L. Jami et al., "Gas-coupled laser acoustic detection as a non-contact line detector for photoacoustic and ultrasound imaging," J. Opt. 18(2), 024005 (2016).

33. R. G. M. Kolkman et al., "Feasibility of noncontact piezoelectric detection of photoacoustic signals in tissue-mimicking phantoms," J. Biomed. Opt. 15(5), 055011 (2010).

34. K. Maslov and L. V. Wang, "Photoacoustic imaging of biological tissue with intensity-modulated continuous-wave laser," J. Biomed. Opt. 13(2), 024006 (2008).

35. A. Petschke and P. J. La Rivière, "Comparison of intensity-modulated continuous-wave lasers with a chirped modulation frequency to pulsed lasers for photoacoustic imaging applications," Biomed. Opt. Express 1(4), 1188-1195 (2010).

36. S. Telenkov et al., "Frequency-domain photothermoacoustics: alternative imaging modality of biological tissues," J. Appl. Phys. 105(10), 102029 (2009).

37. T. Wang et al., "A low-cost photoacoustic microscopy system with a laser diode excitation," Biomed. Opt. Express 5(9), 3053-3058 (2014).

38. K. Sathiyamoorthy, E. M. Strohm, and M. C. Kolios, "Low-power noncontact photoacoustic microscope for bioimaging applications," J. Biomed. Opt. 22(4), 046001 (2017).

39. G. A. West et al., "Photoacoustic spectroscopy," Rev. Sci. Instrum. 54(7), 797-817 (1983).

40. C. Haisch, "Photoacoustic spectroscopy for analytical measurements," Meas. Sci. Technol. 23(1), 012001 (2012).

41. K. Sathiyamoorthy, C. Vijayan, and M. P. Kothiyal, "Simple technique for obtaining photoacoustic spectra corrected for the spectral variation of the source in single scan," Rev. Sci. Instrum. 78(4), 043102 (2007).

42. D. V. Bageshwaret, "Photoacoustic spectroscopy and its applications: a tutorial review," Eurasian J. Anal. Chem. 5, 187-203 (2010).

43. J. Li, W. Chen, and B. Yu, "Recent progress on infrared photoacoustic spectroscopy techniques," Appl. Spectrosc. Rev. 46(6), 440-471 (2011).

44. C. R. Philbrick, "Remote sensing of chemical species in the atmosphere," in Proc. Fourth Symp. Lidar Atmos. Appl., Phoenix, Arizona (2009).

45. T. Kobayashi, "Techniques for laser remote sensing of the environment," Remote Sens. Rev. 3(1), 1-56 (1987).

46. C. M. Wynn et al., "High-sensitivity detection of trace gases using dynamic photoacoustic spectroscopy," Opt. Eng. 53(2), 021103 (2013).

47. A. Elia et al., "Photoacoustic techniques for trace gas sensing based on semiconductor laser sources," Sensors 9(12), 9616-9628 (2009).

48. "SR540- low jitter optical chopper," Stanford Research Systems, Inc., https://www.thinksrs.com/downloads/pdfs/manuals/SR540m.pdf (1997).

49. B. Baumann et al., "Finite element calculation of photoacoustic signals," Appl. Opt. 46(7), 1120-1125 (2007).

50. B. Baumann et al., "Modeling and numerical investigation of photoacoustic resonators," in Recent Advances in Modelling and Simulation, G. Petrone and G. Cammarata, Eds., pp. 17-38, Intech, Europe (2008).

51. Acoustics Module, "COMSOL Multiphysics," https://www.comsol .com/acoustics-module.

52. "Brüel \& Kjær Sound \& Vibration Measurement," https://www.bksv .com/en/microphones and https://www.bksv.com/media/doc/be1447.pdf.

53. Probe Tube microphone and acoustic module, "COMSOL," https:// www.comsol.com/model/probe-tube-microphone-12781 and https:// www.comsol.com/models/acoustics-module.

54. M. C. LoPresto, "Experimenting with end-correction and the speed of sound," Phys. Educ. 46(4), 437-439 (2011).

55. A. D'Alessandro et al., "Red blood cell proteomics update: is there more to discover?" Blood Transfus. 15(2), 182-187 (2017).

Biographies of the authors are not available. 